Yukawa Institute Kyoto

RIMS-924

YITP/K-1018

May 1993

\title{
Free Boson Realization of $U_{q}\left(s \widehat{l}_{N}\right)$
}

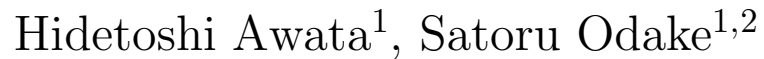 \\ Yukawa Institute for Theoretical Physics \\ Kyoto University, Kyoto 606-01, Japan \\ and \\ Jun'ichi Shiraishi户 \\ Research Institute for Mathematical Sciences \\ Kyoto University, Kyoto 606-01, Japan
}

\begin{abstract}
We construct a realization of the quantum affine algebra $U_{q}\left(\widehat{s l_{N}}\right)$ of an arbitrary level $k$ in terms of free boson fields. In the $q \rightarrow 1$ limit this realization becomes the Wakimoto realization of $\widehat{s l_{N}}$. The screening currents and the vertex operators(primary fields) are also constructed; the former commutes with $U_{q}\left(\widehat{s l_{N}}\right) \bmod$ ulo total difference, and the latter creates the $U_{q}\left(\widehat{s l_{N}}\right)$ highest weight state from the vacuum state of the boson Fock space.

\footnotetext{
${ }^{1}$ Fellow of Soryushi Shogakukai.

${ }^{2}$ Address after June 1; Department of Physics, Faculty of Liberal Arts, Shinshu University, Matsumoto 390, Japan.

${ }^{3}$ On leave from Department of Physics, University of Tokyo, Tokyo 113, Japan.
} 


\section{Introduction}

Chiral algebras such as the Virasoro and current algebras play a central role in the conformal field theory (CFT) in two dimensional space-time. This theory is a quantum field theory (QFT) of massless particles, in other words, a (massive) QFT at a critical point (renormalization-group fixed point)[1]. Perturbing CFT's suitably, we get integrable massive QFT's [8, 3], 4]. In these theories, the Virasoro algebra does not exist any longer. In many cases the quantum affine Lie algebra plays a crucial role instead of the Virasoro algebra [5]. This quantum algebra is, for a large part, at the origin of the integrability. Moreover it can almost determine the S-matrix of the theory, e.g. sine-Gordon model[5].

The Wess-Zumino-Novikov-Witten (WZNW) model is a fundamental example of CFT's; many CFT's can be realized through a coset construction of WZNW models. The WZNW model has been studied based on the representation theory of the affine Lie algebra. Correlation functions of this model, which are vacuum expectation values of vertex operators, satisfy certain holomorphic differential equations, what is called, KnizhnikZamolodchikov (KZ) equations[6, 7]. We expect that " $q$-WZNW model", which has a symmetry of the quantum affine algebra, is certain massive deformation of the WZNW model. Correlation functions of the $q$-WZNW model satisfy $q$-difference equations ( $q$-KZ equations) [8, 9]. Connection matrices of solutions for $q$-KZ equations are related to elliptic solutions of the Yang-Baxter equations of RSOS models [9]. An application of $q$-vertex operators based on $U_{q}\left(\widehat{s l}_{2}\right)$ was performed in diagonalization of the XXZ spin chain 10.

Free field realizations of the Virasoro and affine Lie algebras were useful for studying representation theories 11] and calculating correlation functions 12, 13. It is expected that this is also the case for the quantum affine algebras. In fact, the integral formula for correlation functions of the local operators of the XXZ spin chain was found by using the free boson realization of $U_{q}\left(\widehat{s l}_{2}\right)$ and bosonized $q$-vertex operators 14, 15. To study higher rank versions of the XXZ spin chain, sine-Gordon model, etc., we need free field realizations of the quantum affine algebras.

In this paper we construct a free boson realization of the quantum affine algebra $U_{q}\left(\widehat{s l_{N}}\right)$ with an arbitrary level $k$. In the $q \rightarrow 1$ limit, it becomes the bosonized version of the Wakimoto realization of $\widehat{s l_{N}}$ [16, 17, 18]. Free field realizations of $U_{q}\left(\widehat{s l_{N}}\right)$ with level 1 were constructed in [19]. Free field realizations of $U_{q}\left(\widehat{s l}_{2}\right)$ with an arbitrary level were constructed by several authors [20, 21, 22, 23] and that of $U_{q}\left(\widehat{s l}_{3}\right)$ was obtained by the present authors [24]. We construct a free boson realization of $U_{q}\left(\widehat{s l_{N}}\right)$ by affinizing the Heisenberg realization ( $q$-difference operator realization) of $U_{q}\left(s l_{N}\right)$ [25] and prove it by the OPE (operator product expansion) technique. The screening currents and the vertex operators(primary fields) are also constructed. They are necessary ingredients for calculating correlation functions. Certain integral of the screening current commutes 
with $U_{q}\left(\widehat{s l_{N}}\right)$ and the vertex operator creates the highest weight state of $U_{q}\left(\widehat{s l_{N}}\right)$ from the vacuum state of the boson Fock space.

This paper is organized as follows. In section 2 we fix our notations and recall the definition of $U_{q}\left(\widehat{s l_{N}}\right)$. We construct a free field realization of $U_{q}\left(\widehat{s l_{N}}\right)$ in section 3 , and the screening currents and the vertex operators in section 4. Section 5 is devoted to discussion. The grading operator is also bosonized. In Appendix A we present the Heisenberg realization of $U_{q}\left(s l_{N}\right)$. In Appendix B $q$-difference expressions of our free field realization are given. In Appendix C,D we give useful formulas and some details of calculations.

\section{Notations}

Throughout this paper, complex numbers $q$ and $k$ are fixed. $q$ is assumed to be a generic value with $|q|<1$. We will use the standard symbol $[x]$,

$$
[x] \stackrel{\text { def }}{=} \frac{q^{x}-q^{-x}}{q-q^{-1}},
$$

and $\sum_{r=n}^{n-1} * \stackrel{\text { def }}{=} 0, \prod_{r=n}^{n-1} * \stackrel{\text { def }}{=} 1$. Let $\bar{\alpha}_{i}, \bar{\Lambda}_{i}(1 \leq i \leq N-1),\left(a_{i j}\right)_{1 \leq i, j \leq N-1}$, be the simple roots, fundamental weights, the Cartan matrix of $s l_{N}$ respectively. $(\cdot, \cdot)$ is the symmetric bilinear form; $\left(\bar{\alpha}_{i}, \bar{\alpha}_{j}\right)=a_{i j},\left(\bar{\Lambda}_{i}, \bar{\alpha}_{j}\right)=\delta_{i j} . g$ stands for the dual Coxeter number of $s l_{N}$, i.e., $g=N$.

The $q$-difference operator with a parameter $\alpha$ is defined by [20]

$$
{ }_{\alpha} \partial_{z} f(z) \stackrel{\text { def }}{=} \frac{f\left(q^{\alpha} z\right)-f\left(q^{-\alpha} z\right)}{\left(q-q^{-1}\right) z} .
$$

The Jackson integral with parameters $p \in \mathbb{C}(|p|<1)$ and $s \in \mathbb{C}^{\times}$is defined by

$$
\int_{0}^{s \infty} f(z) d_{p} z \stackrel{\text { def }}{=} s(1-p) \sum_{n \in \mathbb{Z}} f\left(s p^{n}\right) p^{n} .
$$

These operations satisfy the following property:

$$
\int_{0}^{s \infty}{ }_{\alpha} \partial_{z} f(z) d_{p} z=0 \text { for } p=q^{2 \alpha} .
$$

The deformed commutator with a parameter $p \in \mathbb{C}$ is

$$
[A, B]_{p} \stackrel{\text { def }}{=} A B-p B A .
$$

The quantum affine algebra $U_{q}\left(\widehat{s l_{N}}\right)$ is the associative algebra over $\mathbb{C}$ with Chevalley generators $e_{i}^{ \pm}$, invertible $t_{i}(i=0,1, \cdots, N-1)$, and the following relations 26] ${ }^{4}$ :

$$
\left[t_{i}, t_{j}\right]=0
$$

\footnotetext{
${ }^{4}$ For the grading operator $d$, see section 5 .
} 


$$
\begin{aligned}
t_{i} e_{j}^{ \pm} t_{i}^{-1} & =q^{ \pm a_{i j}^{e x t}} e_{j}^{ \pm}, \\
{\left[e_{i}^{+}, e_{j}^{-}\right] } & =\delta_{i j} \frac{t_{i}-t_{i}^{-1}}{q-q^{-1}},
\end{aligned}
$$

and

$$
\sum_{r=0}^{1-a_{i j}^{e x t}}(-1)^{r}\left[\begin{array}{c}
1-a_{i j}^{e x t} \\
r
\end{array}\right]\left(e_{i}^{ \pm}\right)^{1-a_{i j}^{e x t}-r} e_{j}^{ \pm}\left(e_{i}^{ \pm}\right)^{r}=0,
$$

where $\left(a_{i j}^{e x t}\right)_{0 \leq i, j \leq N-1}$ is the Cartan matrix of the extended Dynkin diagram of $s l_{N}$ and $\left[\begin{array}{l}n \\ r\end{array}\right] \stackrel{\text { def }}{=} \frac{[n] !}{[r] ![n-r] !},[n] ! \stackrel{\text { def }}{=} \prod_{r=1}^{n}[r]$.

$U_{q}\left(\widehat{s l_{N}}\right)$ is isomorphic to the associative algebra over $\mathbb{C}$ with Drinfeld generators $E_{n}^{ \pm, i}$ $(n \in \mathbb{Z}), H_{n}^{i}(n \in \mathbb{Z}-\{0\})$, invertible $K_{i}(i=1,2, \cdots, N-1)$, invertible $\gamma$, and the following relations [27]:

$$
\begin{aligned}
\gamma & : \text { central element, } \\
{\left[K_{i}, H_{n}^{j}\right] } & =0, \quad K_{i} E_{n}^{ \pm, j} K_{i}^{-1}=q^{ \pm a_{i j}} E_{n}^{ \pm, j}, \\
{\left[H_{n}^{i}, H_{m}^{j}\right] } & =\frac{1}{n}\left[a_{i j} n\right] \frac{\gamma^{n}-\gamma^{-n}}{q-q^{-1}} \delta_{n+m, 0}, \\
{\left[H_{n}^{i}, E_{m}^{ \pm, j}\right] } & = \pm \frac{1}{n}\left[a_{i j} n\right] \gamma^{\mp \frac{1}{2}|n|} E_{n+m}^{ \pm, j}, \\
{\left[E_{n}^{+, i}, E_{m}^{-, j}\right] } & =\frac{\delta^{i j}}{q-q^{-1}}\left(\gamma^{\frac{1}{2}(n-m)} \psi_{+, n+m}^{i}-\gamma^{-\frac{1}{2}(n-m)} \psi_{-, n+m}^{i}\right),
\end{aligned}
$$

and

$$
\begin{aligned}
& {\left[E_{n+1}^{ \pm, i}, E_{m}^{ \pm, j}\right]_{q^{ \pm a_{i j}}}+\left[E_{m+1}^{ \pm, j}, E_{n}^{ \pm, i}\right]_{q^{ \pm a_{i j}}}=0,} \\
& {\left[E_{n}^{ \pm, i}, E_{m}^{ \pm, j}\right]=0 \text { for } a_{i j}=0,} \\
& {\left[E_{n}^{ \pm, i},\left[E_{m}^{ \pm, i}, E_{\ell}^{ \pm, j}\right]_{q^{\mp 1}}\right]_{q^{ \pm 1}}+\left[E_{m}^{ \pm, i},\left[E_{n}^{ \pm, i}, E_{\ell}^{ \pm, j}\right]_{q^{\mp 1}}\right]_{q^{ \pm 1}}=0 \quad \text { for } a_{i j}=-1 .}
\end{aligned}
$$

Here $\psi_{ \pm, n}^{i}$ are defined by the following equation:

$$
\sum_{n \in \mathbb{Z}} \psi_{ \pm, n}^{i} z^{-n} \stackrel{\text { def }}{=} K_{i}^{ \pm 1} \exp \left( \pm\left(q-q^{-1}\right) \sum_{ \pm n>0} H_{n}^{i} z^{-n}\right)
$$

Let $H_{0}^{i}$ be defined by

$$
K_{i} \stackrel{\text { def }}{=} \exp \left(\left(q-q^{-1}\right) \frac{1}{2} H_{0}^{i}\right),
$$

then eqs.(2.11)-(2.13) hold for $H_{n}^{i}(n \in \mathbb{Z})$. Eq.(2.11) is derived from eqs.(2.12),(2.13).

Defining the fields $H^{i}(z), E^{ \pm, i}(z)$ and $\psi_{ \pm}^{i}(z)$ as

$$
H^{i}(z) \stackrel{\text { def }}{=} \sum_{n \in \mathbb{Z}} H_{n}^{i} z^{-n-1}, \quad E^{ \pm, i}(z) \stackrel{\text { def }}{=} \sum_{n \in \mathbb{Z}} E_{n}^{ \pm, i} z^{-n-1}, \quad \psi_{ \pm}^{i}(z) \stackrel{\text { def }}{=} \sum_{n \in \mathbb{Z}} \psi_{ \pm, n}^{i} z^{-n},
$$

\footnotetext{
${ }^{5}$ In the case of $n=0, \frac{1}{n} *$ should be understood as $\lim _{n \rightarrow 0} \frac{1}{n} *$. For example, $\lim _{n \rightarrow 0} \frac{1}{n}[n]=\frac{2 \log q}{q-q^{-1}}$, $\lim _{n \rightarrow 0} \frac{1}{n}\left[a_{i j} n\right] \frac{\gamma^{n}-\gamma^{-n}}{q-q^{-1}}=0, \lim _{n \rightarrow 0} \frac{1}{n}\left[a_{i j} n\right] \gamma^{\mp \frac{1}{2}|n|}=\frac{2 \log q}{q-q^{-1}} a_{i j}$. In the following, this convention is assumed.
} 
the above relations can be rewritten as formal power series equations:

$$
\begin{aligned}
& {\left[\psi_{ \pm}^{i}(z), \psi_{ \pm}^{j}(w)\right]=0} \\
& \left(z-q^{a_{i j}} \gamma^{-1} w\right)\left(z-q^{-a_{i j}} \gamma w\right) \psi_{+}^{i}(z) \psi_{-}^{j}(w) \\
& \quad=\left(z-q^{a_{i j}} \gamma w\right)\left(z-q^{-a_{i j}} \gamma^{-1} w\right) \psi_{-}^{j}(w) \psi_{+}^{i}(z) \\
& \left(z-q^{ \pm a_{i j}} \gamma^{\mp \frac{1}{2}} w\right) \psi_{+}^{i}(z) E^{ \pm, j}(w)=\left(q^{ \pm a_{i j}} z-\gamma^{\mp \frac{1}{2}} w\right) E^{ \pm, j}(w) \psi_{+}^{i}(z) \\
& \left(z-q^{ \pm a_{i j}} \gamma^{\mp \frac{1}{2}} w\right) E^{ \pm, j}(z) \psi_{-}^{i}(w)=\left(q^{ \pm a_{i j}} z-\gamma^{\mp \frac{1}{2}} w\right) \psi_{-}^{i}(w) E^{ \pm, j}(z), \\
& {\left[E^{+, i}(z), E^{-, j}(w)\right]=\frac{\delta^{i j}}{\left(q-q^{-1}\right) z w}\left(\delta\left(z^{-1} w \gamma\right) \psi_{+}^{i}\left(\gamma^{\frac{1}{2}} w\right)-\delta\left(z^{-1} w \gamma^{-1}\right) \psi_{-}^{i}\left(\gamma^{-\frac{1}{2}} w\right)\right)}
\end{aligned}
$$

and

$$
\begin{aligned}
& \left(z-q^{ \pm a_{i j}} w\right) E^{ \pm, i}(z) E^{ \pm, j}(w)=\left(q^{ \pm a_{i j}} z-w\right) E^{ \pm, j}(w) E^{ \pm, i}(z) \\
& E^{ \pm, i}(z) E^{ \pm, j}(w)=E^{ \pm, j}(w) E^{ \pm, i}(z) \quad \text { for } a_{i j}=0 \\
& E^{ \pm, i}\left(z_{1}\right) E^{ \pm, i}\left(z_{2}\right) E^{ \pm, j}(w)-\left(q+q^{-1}\right) E^{ \pm, i}\left(z_{1}\right) E^{ \pm, j}(w) E^{ \pm, i}\left(z_{2}\right) \\
& \quad+E^{ \pm, j}(w) E^{ \pm, i}\left(z_{1}\right) E^{ \pm, i}\left(z_{2}\right)+\left(\text { replacement }: z_{1} \leftrightarrow z_{2}\right)=0 \quad \text { for } a_{i j}=-1,(2.2
\end{aligned}
$$

where $\delta(x)$ is given by

$$
\delta(x) \stackrel{\text { def }}{=} \sum_{n \in \mathbb{Z}} x^{n} .
$$

Correspondence between Chevalley generators and Drinfeld generators are [27]:

$$
\begin{aligned}
t_{i} & \mapsto K_{i} \quad(i=1, \cdots, N-1), \\
e_{i}^{ \pm} & \mapsto E_{0}^{ \pm, i} \quad(i=1, \cdots, N-1), \\
t_{0} & \mapsto \gamma K_{1}^{-1} \cdots K_{N-1}^{-1}, \\
e_{0}^{+} & \mapsto\left[E_{0}^{-, N-1},\left[E_{0}^{-, N-2},\left[\cdots,\left[E_{0}^{-, 2}, E_{1}^{-, 1}\right]_{q^{-1}} \cdots\right]_{q^{-1}}\right]_{q^{-1}} K_{1}^{-1} \cdots K_{N-1}^{-1},\right. \\
e_{0}^{-} & \mapsto K_{1} \cdots K_{N-1}\left[\left[\cdots\left[E_{-1}^{+, 1}, E_{0}^{+, 2}\right]_{q}, \cdots, E_{0}^{+, N-2}\right]_{q}, E_{0}^{+, N-1}\right]_{q} .
\end{aligned}
$$

$U_{q}\left(\widehat{s l_{N}}\right)$ has the Hopf algebra structure. We take its coproduct $\Delta$ as

$$
\begin{aligned}
\Delta\left(t_{i}\right) & =t_{i} \otimes t_{i}, \\
\Delta\left(e_{i}^{+}\right) & =e_{i}^{+} \otimes 1+t_{i} \otimes e_{i}^{+}, \\
\Delta\left(e_{i}^{-}\right) & =e_{i}^{-} \otimes t_{i}^{-1}+1 \otimes e_{i}^{-},
\end{aligned}
$$

and its antipode $S$ is

$$
S\left(t_{i}\right)=t_{i}^{-1}, \quad S\left(e_{i}^{+}\right)=-t_{i}^{-1} e_{i}^{+}, \quad S\left(e_{i}^{-}\right)=-e_{i}^{-} t_{i} .
$$

An explicit coproduct formula for all the Drinfeld generators has not been obtained. 
Let $V(\lambda)$ be the Verma module over $U_{q}\left(\widehat{s l_{N}}\right)$ generated by the highest weight state $|\lambda\rangle$, such that

$$
\begin{aligned}
H_{n}^{i}|\lambda\rangle & =E_{n}^{ \pm, i}|\lambda\rangle=0 \quad(n>0), \\
E_{0}^{+, i}|\lambda\rangle & =0 \\
H_{0}^{i}|\lambda\rangle & =\ell^{i}|\lambda\rangle,
\end{aligned}
$$

where the classical part of the highest weight is $\bar{\lambda}=\sum_{i=1}^{N-1} \ell^{i} \bar{\Lambda}_{i}$.

Next we will introduce boson fields. For a set of bosonic oscillators $a_{n}(n \in \mathbb{Z})$, and zero modes $\hat{p}_{a}, \hat{q}_{a}$ whose commutation relations are

$$
\begin{array}{rlrl}
{\left[a_{n}, a_{m}\right]} & =n \rho_{a}(n) \delta_{n+m, 0}, & a_{0}=\frac{2 \log q}{q-q^{-1}} \hat{p}_{a}, \\
{\left[\hat{p}_{a}, \hat{q}_{a}\right]} & =\rho_{a}, \quad\left[a_{n}, \hat{q}_{a}\right]=0 \quad(n \neq 0),
\end{array}
$$

where $\rho_{a}$ is a constant and $\rho_{a}(n)$ satisfies

$$
\lim _{q \rightarrow 1} \rho_{a}(n)=\rho_{a}, \quad \lim _{n \rightarrow 0} \rho_{a}(n)=\left(\frac{2 \log q}{q-q^{-1}}\right)^{2} \rho_{a},
$$

we define free boson fields $a(z ; \alpha)$ and $a_{ \pm}(z)$ as follows:

$$
\begin{aligned}
a(z ; \alpha) & \stackrel{\text { def }}{=}-\sum_{n \neq 0} \frac{a_{n}}{[n]} q^{-\alpha|n|} z^{-n}+\hat{q}_{a}+\hat{p}_{a} \log z \\
a_{ \pm}(z) & \stackrel{\text { def }}{=} \pm\left(\left(q-q^{-1}\right) \sum_{ \pm n>0} a_{n} z^{-n}+\hat{p}_{a} \log q\right) \\
& = \pm\left(q-q^{-1}\right)\left(\sum_{ \pm n>0} a_{n} z^{-n}+\frac{1}{2} a_{0}\right) .
\end{aligned}
$$

We abbreviate $a(z ; 0)$ as $a(z) \stackrel{\text { def }}{=} a(z ; 0)$. In the $q \rightarrow 1$ limit $a(z ; \alpha)$ becomes the free chiral boson field $\phi(z)$ used in the string theory and CFT (but the meaning of $z$ is different). Correspondence between $a(z ; \alpha)$ and $\phi(z)=\hat{x}-\sqrt{-1} \hat{p} \log z+\sqrt{-1} \sum_{n \neq 0} \frac{1}{n} \alpha_{n} z^{-n}$ is

$$
a(z ; \alpha) \rightarrow \sqrt{-1} \sqrt{\rho_{a}} \phi(z), \quad a_{n} \rightarrow \sqrt{\rho_{a}} \alpha_{n}, \quad \hat{p}_{a} \rightarrow \sqrt{\rho_{a}} \hat{p}, \quad \hat{q}_{a} \rightarrow \sqrt{-1} \sqrt{\rho_{a}} \hat{x} .
$$

Moreover let us define boson fields with parameters $L, M$ as follows:

$$
\begin{aligned}
& a\left(L_{1}, \cdots, L_{r} ; M_{1}, \cdots, M_{r} \mid z ; \alpha\right) \\
& \stackrel{\text { def }}{=}-\sum_{n \neq 0} \frac{\left[L_{1} n\right] \cdots\left[L_{r} n\right]}{\left[M_{1} n\right] \cdots\left[M_{r} n\right]} \frac{a_{n}}{[n]} q^{-\alpha|n|} z^{-n}+\frac{L_{1} \cdots L_{r}}{M_{1} \cdots M_{r}}\left(\hat{q}_{a}+\hat{p}_{a} \log z\right), \\
& a_{ \pm}\left(L_{1}, \cdots, L_{r} ; M_{1}, \cdots, M_{r} \mid z\right) \\
& \stackrel{\text { def }}{=} \pm\left(\left(q-q^{-1}\right) \sum_{ \pm n>0} \frac{\left[L_{1} n\right] \cdots\left[L_{r} n\right]}{\left[M_{1} n\right] \cdots\left[M_{r} n\right]} a_{n} z^{-n}+\frac{L_{1} \cdots L_{r}}{M_{1} \cdots M_{r}} \hat{p}_{a} \log q\right) \\
& = \pm\left(q-q^{-1}\right)\left(\sum_{ \pm n>0} \frac{\left[L_{1} n\right] \cdots\left[L_{r} n\right]}{\left[M_{1} n\right] \cdots\left[M_{r} n\right]} a_{n} z^{-n}+\frac{L_{1} \cdots L_{r}}{M_{1} \cdots M_{r}} \frac{1}{2} a_{0}\right) .
\end{aligned}
$$


We abbreviate these as

$$
\begin{gathered}
\left(\frac{L_{1}}{M_{1}} \frac{L_{2}}{M_{2}} \cdots \frac{L_{r}}{M_{r}} a\right)(z ; \alpha) \stackrel{\text { def }}{=} a\left(L_{1}, L_{2}, \cdots, L_{r} ; M_{1}, M_{2}, \cdots, M_{r} \mid z ; \alpha\right), \\
\left(\frac{L_{1}}{M_{1}} \frac{L_{2}}{M_{2}} \cdots \frac{L_{r}}{M_{r}} a_{ \pm}\right)(z) \stackrel{\text { def }}{=} a_{ \pm}\left(L_{1}, L_{2}, \cdots, L_{r} ; M_{1}, M_{2}, \cdots, M_{r} \mid z\right) .
\end{gathered}
$$

Normal ordering prescription : : is defined by

$$
\left\{\begin{array}{lll}
\text { move } & a_{n}(n>0) \text { and } \hat{p}_{a} & \text { to right } \\
\text { move } & a_{n}(n<0) \text { and } \hat{q}_{a} & \text { to left. }
\end{array}\right.
$$

For example,

$$
: \exp (a(z ; \alpha)):=\exp \left(-\sum_{n<0} \frac{a_{n}}{[n]}\left(q^{-\alpha} z\right)^{-n}\right) e^{\hat{q}_{a}} z^{\hat{p}_{a}} \exp \left(-\sum_{n>0} \frac{a_{n}}{[n]}\left(q^{\alpha} z\right)^{-n}\right) .
$$

For multicomponent $a^{i}\left(a_{n}^{i}, \hat{p}_{a}^{i}, \hat{q}_{a}^{i}\right)$, we treat them similarly; $\left[a_{n}^{i}, a_{m}^{j}\right]=n \rho_{a}^{i j}(n) \delta_{n+m, 0}$, etc. We can easily verify the following:

$$
\left[\frac{1}{2} \sum_{i, j} \sum_{n \in \mathbb{Z}}: a_{-n}^{i} \rho_{a}^{-1, i j}(n) a_{n}^{j}:, a_{m}^{\ell}\right]=-m a_{m}^{\ell}
$$

where $\rho_{a}^{-1, i j}(n)$ is an inverse of $\rho_{a}^{i j}(n)$, i.e., $\sum_{\ell} \rho_{a}^{i \ell}(n) \rho_{a}^{-1, \ell j}(n)=\delta^{i j}$.

\section{Free Boson Realization of $U_{q}\left(\widehat{s l}_{N}\right)$}

To construct the Drinfeld $U_{q}\left(\widehat{s l_{N}}\right)$ generators of level $k$ in terms of free boson fields, we need $N^{2}-1$ free boson fields $a^{i}(1 \leq i \leq N-1), b^{i j}$ and $c^{i j}(1 \leq i<j \leq N)$. Their commutation relations are

$$
\begin{array}{rlrl}
{\left[a_{n}^{i}, a_{m}^{j}\right]} & =\frac{1}{n}[(k+g) n]\left[a_{i j} n\right] \delta_{n+m, 0}, & {\left[\hat{p}_{a}^{i}, \hat{q}_{a}^{j}\right]=(k+g) a_{i j},} \\
{\left[b_{n}^{i j}, b_{m}^{i^{\prime} j^{\prime}}\right]=-\frac{1}{n}[n]^{2} \delta^{i i^{\prime}} \delta^{j j^{\prime}} \delta_{n+m, 0},} & {\left[\hat{p}_{b}^{i j}, \hat{q}_{b}^{i^{\prime} j^{\prime}}\right]=-\delta^{i i^{\prime}} \delta^{j j^{\prime}},} \\
{\left[c_{n}^{i j}, c_{m}^{i^{\prime} j^{\prime}}\right]=\frac{1}{n}[n]^{2} \delta^{i i^{\prime}} \delta^{j j^{\prime}} \delta_{n+m, 0},} & {\left[\hat{p}_{c}^{i j}, \hat{q}_{c}^{i^{\prime} j^{\prime}}\right]=\delta^{i i^{\prime}} \delta^{j j^{\prime}},}
\end{array}
$$

and the remaining commutators vanish.

Let us define fields $H^{i}(z), \psi_{ \pm}^{i}(z)$ and $E^{ \pm, i}(z)(1 \leq i \leq N-1)$ as follows ${ }^{6}$ :

$$
\begin{aligned}
H^{i}(z) \stackrel{\text { def }}{=} & \frac{1}{\left(q-q^{-1}\right) z} \\
& \times\left(\sum_{j=1}^{i}\left(b_{+}^{j, i+1}\left(q^{\frac{k}{2}+j-1} z\right)-b_{+}^{j, i}\left(q^{\frac{k}{2}+j} z\right)\right)\right.
\end{aligned}
$$

${ }^{6}$ These operators are well-defined on the boson Fock space that will be defined in the next section. 


$$
\begin{aligned}
& \left.+a_{+}^{i}\left(q^{\frac{g}{2}} z\right)+\sum_{j=i+1}^{N}\left(b_{+}^{i, j}\left(q^{\frac{k}{2}+j} z\right)-b_{+}^{i+1, j}\left(q^{\frac{k}{2}+j-1} z\right)\right)\right) \\
& -\left(\text { replacement }: x_{+}\left(q^{\alpha} z\right) \mapsto x_{-}\left(q^{-\alpha} z\right) \text { for } x=a, b\right), \\
& \psi_{ \pm}^{i}\left(q^{ \pm \frac{k}{2}} z\right) \stackrel{\text { def }}{=}: \exp \left(\sum_{j=1}^{i}\left(b_{ \pm}^{j, i+1}\left(q^{ \pm(k+j-1)} z\right)-b_{ \pm}^{j, i}\left(q^{ \pm(k+j)} z\right)\right)\right. \\
& \left.+a_{ \pm}^{i}\left(q^{ \pm \frac{k+g}{2}} z\right)+\sum_{j=i+1}^{N}\left(b_{ \pm}^{i, j}\left(q^{ \pm(k+j)} z\right)-b_{ \pm}^{i+1, j}\left(q^{ \pm(k+j-1)} z\right)\right)\right): \\
& E^{+, i}(z) \stackrel{\text { def }}{=} \frac{-1}{\left(q-q^{-1}\right) z} \\
& \times \sum_{j=1}^{i}: \exp \left((b+c)^{j, i}\left(q^{j-1} z\right)\right) \\
& \times\left(\exp \left(b_{+}^{j, i+1}\left(q^{j-1} z\right)-(b+c)^{j, i+1}\left(q^{j} z\right)\right)\right. \\
& \left.-\exp \left(b_{-}^{j, i+1}\left(q^{j-1} z\right)-(b+c)^{j, i+1}\left(q^{j-2} z\right)\right)\right) \\
& \times \exp \left(\sum_{\ell=1}^{j-1}\left(b_{+}^{\ell, i+1}\left(q^{\ell-1} z\right)-b_{+}^{\ell, i}\left(q^{\ell} z\right)\right)\right): \\
& E^{-, i}(z) \stackrel{\text { def }}{=} \frac{-1}{\left(q-q^{-1}\right) z} \\
& \times\left(\sum_{j=1}^{i-1}: \exp \left((b+c)^{j, i+1}\left(q^{-(k+j)} z\right)\right)\right. \\
& \times\left(\exp \left(-b_{-}^{j, i}\left(q^{-(k+j)} z\right)-(b+c)^{j, i}\left(q^{-(k+j-1)} z\right)\right)\right. \\
& \left.-\exp \left(-b_{+}^{j, i}\left(q^{-(k+j)} z\right)-(b+c)^{j, i}\left(q^{-(k+j+1)} z\right)\right)\right) \\
& \times \exp \left(\sum_{\ell=j+1}^{i}\left(b_{-}^{\ell, i+1}\left(q^{-(k+\ell-1)} z\right)-b_{-}^{\ell, i}\left(q^{-(k+\ell)} z\right)\right)\right. \\
& \left.+a_{-}^{i}\left(q^{-\frac{k+g}{2}} z\right)+\sum_{\ell=i+1}^{N}\left(b_{-}^{i, \ell}\left(q^{-(k+\ell)} z\right)-b_{-}^{i+1, \ell}\left(q^{-(k+\ell-1)} z\right)\right)\right): \\
& +: \exp \left((b+c)^{i, i+1}\left(q^{-(k+i)} z\right)\right) \\
& \times \exp \left(a_{-}^{i}\left(q^{-\frac{k+g}{2}} z\right)+\sum_{\ell=i+1}^{N}\left(b_{-}^{i, \ell}\left(q^{-(k+\ell)} z\right)-b_{-}^{i+1, \ell}\left(q^{-(k+\ell-1)} z\right)\right)\right): \\
& -: \exp \left((b+c)^{i, i+1}\left(q^{k+i} z\right)\right) \\
& \times \exp \left(a_{+}^{i}\left(q^{\frac{k+g}{2}} z\right)+\sum_{\ell=i+1}^{N}\left(b_{+}^{i, \ell}\left(q^{k+\ell} z\right)-b_{+}^{i+1, \ell}\left(q^{k+\ell-1} z\right)\right)\right): \\
& -\sum_{j=i+2}^{N}: \exp \left((b+c)^{i, j}\left(q^{k+j-1} z\right)\right) \\
& \times\left(\exp \left(b_{+}^{i+1, j}\left(q^{k+j-1} z\right)-(b+c)^{i+1, j}\left(q^{k+j} z\right)\right)\right.
\end{aligned}
$$




$$
\begin{gathered}
\left.-\exp \left(b_{-}^{i+1, j}\left(q^{k+j-1} z\right)-(b+c)^{i+1, j}\left(q^{k+j-2} z\right)\right)\right) \\
\left.\times \exp \left(a_{+}^{i}\left(q^{\frac{k+g}{2}} z\right)+\sum_{\ell=j}^{N}\left(b_{+}^{i, \ell}\left(q^{k+\ell} z\right)-b_{+}^{i+1, \ell}\left(q^{k+\ell-1} z\right)\right)\right):\right),
\end{gathered}
$$

where $b^{i i} \stackrel{\text { def }}{=} 0, c^{i i} \stackrel{\text { def }}{=} 0$ and $(b+c)^{i j} \stackrel{\text { def }}{=} b^{i j}+c^{i j}$. These expressions are guessed from free boson realizations of $U_{q}\left(\widehat{s l_{2}}\right)$ [20], $U_{q}\left(\widehat{s l_{3}}\right)$ [24] and the Heisenberg realization of $U_{q}\left(s l_{N}\right)$ 25] (Appendix A). $q$-difference expressions of these fields are given in Appendix B. In the $q \rightarrow 1$ limit, eqs. 3.4), (3.6) and (3.7) become the bosonized version of the Wakimoto realization of $\widehat{s l_{N}}$ with level $k$ 16, 17, 18.

From eqs.(3.4) and (2.19), $H_{n}^{i}$ and $K_{i}$ are

$$
\begin{aligned}
H_{n}^{i}= & \sum_{j=1}^{i}\left(b_{n}^{j, i+1} q^{-\left(\frac{k}{2}+j-1\right)|n|}-b_{n}^{j, i} q^{-\left(\frac{k}{2}+j\right)|n|}\right) \\
& +a_{n}^{i} q^{-\frac{g}{2}|n|}+\sum_{j=i+1}^{N}\left(b_{n}^{i, j} q^{-\left(\frac{k}{2}+j\right)|n|}-b_{n}^{i+1, j} q^{-\left(\frac{k}{2}+j-1\right)|n|}\right), \\
K_{i}= & q^{\sum_{j=1}^{i}\left(\hat{p}_{b}^{j, i+1}-\hat{p}_{b}^{j, i}\right)+\hat{p}_{a}^{i}+\sum_{j=i+1}^{N}\left(\hat{p}_{b}^{i, j}-\hat{p}_{b}^{i+1, j}\right)} .
\end{aligned}
$$

We obtain the following proposition:

Proposition $1 H^{i}, \psi_{ \pm}^{i}, E^{ \pm, i}$ in eqs.(3.4)-(3.X) satisfy the relations eqs.(2.10)-(2.13) with $\gamma=q^{k}$, eq. 2.28), and the following relations:

$$
\begin{aligned}
& E^{+, i}(z) E^{-, j}(w) \simeq E^{-, j}(w) E^{+, i}(z) \\
& \quad \sim \operatorname{reg} .+\frac{\delta^{i j}}{\left(q-q^{-1}\right) w}\left(\frac{1}{z-q^{k} w} \psi_{+}^{i}\left(q^{\frac{k}{2}} w\right)-\frac{1}{z-q^{-k} w} \psi_{-}^{i}\left(q^{-\frac{k}{2}} w\right)\right), \\
& \left(z-q^{ \pm a_{i j}} w\right) E^{ \pm, i}(z) E^{ \pm, j}(w) \simeq\left(q^{ \pm a_{i j}} z-w\right) E^{ \pm, j}(w) E^{ \pm, i}(z) \sim \text { reg. } \\
& E^{ \pm, i}(z) E^{ \pm, j}(w) \simeq E^{ \pm, j}(w) E^{ \pm, i}(z) \sim \text { reg. } \quad \text { for } a_{i j}=0,
\end{aligned}
$$

where the symbol $\simeq$ and $\sim$ mean equality in the OPE sense (in other words analytic continuation sense), and $\sim$ means equality modulo regular parts.

Proof. A straightforward but tedious OPE calculation shows this proposition. We give the useful formulas in Appendix $\mathrm{C}$ and how the poles cancel each other in Appendix D. For eq.(2.28) some explanation is needed. Let us denote OPE of each term of $E^{ \pm, i}(z)$ as follows (see Appendix D for notation):

$$
E^{ \pm, i(A)}(z) E^{ \pm, j(B)}(w) \simeq f_{ \pm}^{i j A B}(z, w): E^{ \pm, i(A)}(z) E^{ \pm, j(B)}(w):
$$

For $i=j$ there are three cases:

$$
f_{ \pm}^{i i A B}(z, w)=q^{\ell} \frac{z-w}{z-q^{ \pm 2} w} \text { and } f_{ \pm}^{i i B A}(w, z)=q^{\ell} \frac{w-z}{w-q^{ \pm 2} z}
$$




$$
\begin{aligned}
& f_{ \pm}^{i i A B}(z, w)=q^{\ell} \frac{q^{ \pm 2} z-w}{z-q^{ \pm 2} w} \text { and } f_{ \pm}^{i i B A}(w, z)=q^{\ell} \\
& f_{ \pm}^{i i A B}(z, w)=q^{\ell} \text { and } f_{ \pm}^{i i B A}(w, z)=q^{\ell} \frac{q^{ \pm 2} w-z}{w-q^{ \pm 2} z}
\end{aligned}
$$

where $\ell \in \mathbb{Z}$ depends on $i, j, A, B, \pm$. For $a_{i j}=-1$ there are two casest:

$$
\begin{aligned}
& f_{ \pm}^{i j A B}(z, w)=q^{m} \frac{q^{\mp 1} z-w}{z-q^{\mp 1} w} \text { and } f_{ \pm}^{j i B A}(w, z)=q^{m} \\
& f_{ \pm}^{i j A B}(z, w)=q^{m} \text { and } f_{ \pm}^{j i B A}(w, z)=q^{m} \frac{q^{\mp 1} w-z}{w-q^{\mp 1} z}
\end{aligned}
$$

where $m \in \mathbb{Z}$ depends on $i, j, A, B, \pm$. These OPE equations can be translated to formal power series equations:

$$
E^{ \pm, i(A)}(z) E^{ \pm, j(B)}(w)=g_{ \pm}^{i j A B}(z, w): E^{ \pm, i(A)}(z) E^{ \pm, j(B)}(w):
$$

Eqs.(3.14)-(3.18) are translated to

$$
\begin{aligned}
g_{ \pm}^{i i A B}(z, w)= & q^{\ell}(z-w) \frac{1}{z} \sum_{n \geq 0}\left(q^{ \pm 2} \frac{w}{z}\right)^{n} \\
\text { and } \quad & g_{ \pm}^{i i B A}(w, z)=q^{\ell}(w-z) \frac{1}{w} \sum_{n \geq 0}\left(q^{ \pm 2} \frac{z}{w}\right)^{n} \\
g_{ \pm}^{i i A B}(z, w)= & q^{\ell}\left(q^{ \pm 2} z-w\right) \frac{1}{z} \sum_{n \geq 0}\left(q^{ \pm 2} \frac{w}{z}\right)^{n} \text { and } g_{ \pm}^{i i B A}(w, z)=q^{\ell} \\
g_{ \pm}^{i i A B}(z, w)= & q^{\ell} \text { and } g_{ \pm}^{i i B A}(w, z)=q^{\ell}\left(q^{ \pm 2} w-z\right) \frac{1}{w} \sum_{n \geq 0}\left(q^{ \pm 2} \frac{z}{w}\right)^{n} \\
g_{ \pm}^{i j A B}(z, w)= & q^{m}\left(q^{\mp 1} z-w\right) \frac{1}{z} \sum_{n \geq 0}\left(q^{\mp 1} \frac{w}{z}\right)^{n} \text { and } g_{ \pm}^{j i B A}(w, z)=q^{m} \\
g_{ \pm}^{i j A B}(z, w)= & q^{m} \text { and } g_{ \pm}^{j i B A}(w, z)=q^{m}\left(q^{\mp 1} w-z\right) \frac{1}{w} \sum_{n \geq 0}\left(q^{\mp 1} \frac{z}{w}\right)^{n}
\end{aligned}
$$

respectively. A product of three $E$ 's can be expressed as

$$
\begin{aligned}
& E^{ \pm, i_{1}\left(A_{1}\right)}\left(z_{1}\right) E^{ \pm, i_{2}\left(A_{2}\right)}\left(z_{2}\right) E^{ \pm, i_{3}\left(A_{3}\right)}\left(z_{3}\right) \\
= & g_{ \pm}^{i_{1} i_{2} A_{1} A_{2}}\left(z_{1}, z_{2}\right) g_{ \pm}^{i_{1} i_{3} A_{1} A_{3}}\left(z_{1}, z_{3}\right) g_{ \pm}^{i_{2} i_{3} A_{2} A_{3}}\left(z_{2}, z_{3}\right) \\
& \times: E^{ \pm, i_{1}\left(A_{1}\right)}\left(z_{1}\right) E^{ \pm, i_{2}\left(A_{2}\right)}\left(z_{2}\right) E^{ \pm, i_{3}\left(A_{3}\right)}\left(z_{3}\right):
\end{aligned}
$$

We remark that this is a consequence of the bosonic realization. Using this fact, we obtain

$$
\begin{aligned}
& E^{ \pm, i\left(A_{1}\right)}\left(z_{1}\right) E^{ \pm, i\left(A_{2}\right)}\left(z_{2}\right) E^{ \pm, j(B)}(w)-\left(q+q^{-1}\right) E^{ \pm, i\left(A_{1}\right)}\left(z_{1}\right) E^{ \pm, j(B)}(w) E^{ \pm, i\left(A_{2}\right)}\left(z_{2}\right) \\
& +E^{ \pm, j(B)}(w) E^{ \pm, i\left(A_{1}\right)}\left(z_{1}\right) E^{ \pm, i\left(A_{2}\right)}\left(z_{2}\right) \\
= & g_{ \pm}^{i i A_{1} A_{2}}\left(z_{1}, z_{2}\right)\left(g_{ \pm}^{i j A_{1} B}\left(z_{1}, w\right) g_{ \pm}^{i j A_{2} B}\left(z_{2}, w\right)-\left(q+q^{-1}\right) g_{ \pm}^{i j A_{1} B}\left(z_{1}, w\right) g_{ \pm}^{j i B A_{2}}\left(w, z_{2}\right)\right. \\
& \left.\quad+g_{ \pm}^{j i B A_{1}}\left(w, z_{1}\right) g_{ \pm}^{j i B A_{2}}\left(w, z_{2}\right)\right) \times: E^{ \pm, i\left(A_{1}\right)}\left(z_{1}\right) E^{ \pm, i\left(A_{2}\right)}\left(z_{2}\right) E^{ \pm, j(B)}(w):
\end{aligned}
$$

\footnotetext{
7 For $E^{-}$, there are extra poles. However, we can discard them because they cancel each other.
} 
In each case, this coefficient is antisymmetric with respect to $z_{1}$ and $z_{2}$. Therefore eq.(2.28) holds.

We remark that eqs.(3.10), (3.11), (3.12) imply eqs.(2.25), (2.26), (2.27) respectively. Therefore we obtain our main statement:

Corollary $2 H^{i}, \psi_{ \pm}^{i}, E^{ \pm, i}$ in eqs.(3.4)-(3.X) realize the quantum affine algebra $U_{q}\left(\widehat{s l_{N}}\right)$ in the Drinfeld realization with $\gamma=q^{k}$.

\section{Screening Currents and Vertex Operators}

To calculate correlation functions and investigate the irreducible representation, we need screening operators, which commute with $U_{q}\left(\widehat{s l_{N}}\right)$. Let us define the screening currents $S^{i}(z)(i=1, \cdots, N-1)$ as follows:

$$
\begin{aligned}
& S^{i}(z) \stackrel{\text { def }}{=}: \exp \left(-\left(\frac{1}{k+g} a^{i}\right)\left(z ; \frac{k+g}{2}\right)\right): \tilde{S}^{i}(z) \\
& \tilde{S}^{i}(z) \stackrel{\text { def }}{=} \frac{-1}{\left(q-q^{-1}\right) z} \\
& \times \sum_{j=i+1}^{N} \quad \exp \left((b+c)^{i+1, j}\left(q^{N-j} z\right)\right) \\
& \quad\left(\exp \left(-b_{-}^{i, j}\left(q^{N-j} z\right)-(b+c)^{i, j}\left(q^{N-j+1} z\right)\right)\right. \\
&\left.\quad-\exp \left(-b_{+}^{i, j}\left(q^{N-j} z\right)-(b+c)^{i, j}\left(q^{N-j-1} z\right)\right)\right) \\
& \quad \times \exp \left(\sum_{\ell=j+1}^{N}\left(b_{-}^{i+1, \ell}\left(q^{N-\ell+1} z\right)-b_{-}^{i, \ell}\left(q^{N-\ell} z\right)\right)\right): .
\end{aligned}
$$

We remark that $\tilde{S}^{i}(z)$ is nothing else but $E^{+, N-i}(z)$ with replacement $b_{ \pm}^{i, j} \mapsto-b_{\mp}^{N+1-j, N+1-i}$, $(b+c)^{i, j} \mapsto(b+c)^{N+1-j, N+1-i}$. These screening currents have the following properties.

Proposition $3 S^{i}, \tilde{S}^{i}$ in eqs.(4.1), (4.9) and $H^{i}, E^{ \pm, i}$ in eqs.(3.4)-(3.7) satisfy the following relations:

$$
\begin{aligned}
{\left[H_{n}^{i}, S^{j}(z)\right] } & =0, \\
E^{+, i}(z) S^{j}(w) & \simeq S^{j}(w) E^{+, i}(z) \sim \text { reg. } \\
E^{-, i}(z) S^{j}(w) & \simeq S^{j}(w) E^{-, i}(z) \\
& \sim \text { reg. }+\delta_{k+g}^{i j} \partial_{w}\left(\frac{1}{z-w}: \exp \left(-\left(\frac{1}{k+g} a^{j}\right)\left(w ;-\frac{k+g}{2}\right)\right):\right),
\end{aligned}
$$

and

$$
\begin{aligned}
& \left(z-q^{-a_{i j}} w\right) \tilde{S}^{i}(z) \tilde{S}^{j}(w) \simeq\left(q^{-a_{i j}} z-w\right) \tilde{S}^{j}(w) \tilde{S}^{i}(z) \sim \text { reg. } \\
& \tilde{S}^{i}(z) \tilde{S}^{j}(w) \simeq \tilde{S}^{j}(w) \tilde{S}^{i}(z) \sim \text { reg. } \quad \text { for } a_{i j}=0 .
\end{aligned}
$$


Proof. Straightforward (see Appendices C,D).

Eqs.(4.3)-(4.5) can be expressed in the commutator form.

\section{Corollary 4}

$$
\begin{aligned}
{\left[H_{n}^{i}, S^{j}(z)\right] } & =0, \\
{\left[E_{n}^{+, i}, S^{j}(z)\right] } & =0, \\
{\left[E_{n}^{-, i}, S^{j}(z)\right] } & =\delta^{i j}{ }_{k+g} \partial_{z}\left(z^{n}: \exp \left(-\left(\frac{1}{k+g} a^{j}\right)\left(z ;-\frac{k+g}{2}\right)\right):\right) .
\end{aligned}
$$

From this we get the desired property of the screening charges.

Corollary 5 If the Jackson integrals of the screening currents eq.(4.1),

$$
\int_{0}^{s \infty} S^{i}(z) d_{p} z, \quad p=q^{2(k+g)}
$$

are convergent, they commute with $U_{q}\left(\widehat{s l_{N}}\right)$ generated by eqs.(3.4)-(3.7).

Next we will construct the vertex operators(primary fields), which create the $U_{q}\left(\widehat{s l_{N}}\right)$ highest weight states from the vacuum state of the boson Fock space. The vacuum state of the boson Fock space, $|\mathbf{0}\rangle$, is defined by

$$
a_{n}^{i}|\mathbf{0}\rangle=b_{n}^{i j}|\mathbf{0}\rangle=c_{n}^{i j}|\mathbf{0}\rangle=0 \quad(n \geq 0) .
$$

Let $\left|p_{a}, p_{b}, p_{c}\right\rangle$ be

$$
\left|p_{a}, p_{b}, p_{c}\right\rangle \stackrel{\text { def }}{=} \exp \left(\sum_{i, j=1}^{N-1} p_{a}^{i} \frac{a_{i j}^{-1}}{k+g} \hat{q}_{a}^{j}+\sum_{1 \leq i<j \leq N} p_{b}^{i j}(-1) \hat{q}_{b}^{i j}+\sum_{1 \leq i<j \leq N} p_{c}^{i j} \hat{q}_{c}^{i j}\right)|\mathbf{0}\rangle
$$

then $\left|p_{a}, p_{b}, p_{c}\right\rangle$ is the highest weight state of the boson Fock space, i.e.,

$$
\begin{aligned}
& a_{n}^{i}\left|p_{a}, p_{b}, p_{c}\right\rangle=b_{n}^{i j}\left|p_{a}, p_{b}, p_{c}\right\rangle=c_{n}^{i j}\left|p_{a}, p_{b}, p_{c}\right\rangle=0 \quad(n>0), \\
& \hat{p}_{a}^{i}\left|p_{a}, p_{b}, p_{c}\right\rangle=p_{a}^{i}\left|p_{a}, p_{b}, p_{c}\right\rangle, \quad \hat{p}_{x}^{i j}\left|p_{a}, p_{b}, p_{c}\right\rangle=p_{x}^{i j}\left|p_{a}, p_{b}, p_{c}\right\rangle \quad(x=b, c) .
\end{aligned}
$$

The boson Fock space $F\left(p_{a}, p_{b}, p_{c}\right)$ is generated by oscillators of negative mode on the highest weight state $\left|p_{a}, p_{b}, p_{c}\right\rangle . E_{n}^{ \pm, i}$ change $p_{b}-p_{c}$ only, $S_{n}^{i}$ changes $p_{a}$ and $p_{b}-p_{c}, H_{n}^{i}$ does not change $p_{a}, p_{b}, p_{c} .\left|p_{a}, 0,0\right\rangle$ has the following property:

Proposition $6 \mathrm{H}^{i}, E^{ \pm, i}$ in eqs.(3.4)-(3.X) act on $\left|p_{a}, 0,0\right\rangle$ as follows:

$$
\begin{aligned}
X_{n}\left|p_{a}, 0,0\right\rangle & =0 \quad\left(n>0 ; X=H^{i}, E^{ \pm, i}\right), \\
E_{0}^{+, i}\left|p_{a}, 0,0\right\rangle & =0 \\
H_{0}^{i}\left|p_{a}, 0,0\right\rangle & =p_{a}^{i}\left|p_{a}, 0,0\right\rangle .
\end{aligned}
$$


Proof. Straightforward. $X_{n}(n>0)$ annihilate $\left|p_{a}, p_{b}, p_{c}\right\rangle$ with $p_{b}+p_{c}=0$, and $E_{0}^{+, i}$ annihilate $\left|p_{a}, 0,0\right\rangle$.

This property is just the highest weight state condition of $U_{q}\left(\widehat{s l_{N}}\right)$.

Corollary 7 Using the highest weight state $\left|p_{a}, 0,0\right\rangle=\left|\left(\ell^{1}, \cdots, \ell^{N-1}\right), 0,0\right\rangle$, we get the highest weight left module of $U_{q}\left(\widehat{s l_{N}}\right), V(\lambda)$,

$$
V(\lambda) \hookrightarrow \bigoplus_{r \in \mathbb{Z}^{N(N-1) / 2}} F\left(\left(\ell^{1}, \cdots, \ell^{N-1}\right), r,-r\right),
$$

where the classical part of the highest weight is $\bar{\lambda}=\ell^{1} \bar{\Lambda}_{1}+\cdots+\ell^{N-1} \bar{\Lambda}_{N-1}=\left(\ell^{1}, \cdots, \ell^{N-1}\right)$.

As is well known in CFT, this module is reducible.

Let us define the vertex operator with a weight $\bar{\lambda}=\left(\ell^{1}, \cdots, \ell^{N-1}\right)$ and a parameter $\alpha$, $\phi^{\bar{\lambda}}(z ; \alpha)$, as follows:

$$
\phi^{\bar{\lambda}}(z ; \alpha) \stackrel{\text { def }}{=}: \exp \left(\sum_{i, j=1}^{N-1}\left(\frac{\ell^{i}}{k+g} \frac{\min (i, j)}{N} \frac{N-\max (i, j)}{1} a^{j}\right)(z ; \alpha)\right): .
$$

The highest weight state of $U_{q}\left(\widehat{s l_{N}}\right),\left|\left(\ell^{1}, \cdots, \ell^{N-1}\right), 0,0\right\rangle$, is created from the vacuum $|\mathbf{0}\rangle$ by this operator with any parameters $\alpha$ and $\beta$,

$$
\left|\left(\ell^{1}, \cdots, \ell^{N-1}\right), 0,0\right\rangle=\lim _{z \rightarrow 0} \phi^{\bar{\lambda}}\left(q^{\beta} z ; \alpha\right)|\mathbf{0}\rangle .
$$

Moreover this vertex operator has the following properties.

Proposition $8 \phi^{\bar{\lambda}}$ in eq.(4.2Q) and $H^{i}, E^{ \pm, i}$ in eqs.(3.4)-(3.7) satisfy the following relations:

$$
\begin{aligned}
{\left[H_{n}^{i}, \phi^{\bar{\lambda}}(z ; \alpha)\right] } & =\frac{1}{n}\left[\ell^{i} n\right] q^{-\left(\alpha+\frac{g}{2}\right)|n|} z^{n} \phi^{\bar{\lambda}}(z ; \alpha), \\
{\left[E_{n}^{+, i}, \phi^{\bar{\lambda}}(z ; \alpha)\right] } & =0
\end{aligned}
$$

and

$$
\left(z-q^{\ell^{i}} w\right) E^{-, i}(z) \phi^{\bar{\lambda}}\left(w ;-\frac{k+g}{2}\right) \simeq\left(q^{\ell^{i}} z-w\right) \phi^{\bar{\lambda}}\left(w ;-\frac{k+g}{2}\right) E^{-, i}(z) \sim \text { reg. }
$$

Proof. Straightforward. We use the $q$-analogue of the inverse of the Cartan matrix:

$$
\sum_{r=1}^{N-1} \frac{\left[a_{i r} n\right]}{[n]} \frac{[\min (r, j) n][(N-\max (r, j)) n]}{[N n][n]}=\delta_{i j} .
$$

We remark that eq.4.24) can be rewritten as

$$
\left[E_{n}^{-, i}, \phi^{\bar{\lambda}}\left(z ;-\frac{k+g}{2}\right)\right]_{q^{\ell^{i}}}=-z\left[\phi^{\bar{\lambda}}\left(z ;-\frac{k+g}{2}\right), E_{n-1}^{-, i}\right]_{q^{\ell^{i}}} .
$$

From $\phi^{\bar{\lambda}}\left(q^{\beta} z ; \alpha\right)$ with appropriate $\alpha$ and $\beta$, we can construct the $q$-vertex operator $\Phi(z)$ 9], which has an intertwining property. We will discuss this problem in the next section. 


\section{Discussion}

In this paper we have constructed a free boson realization of $U_{q}\left(\widehat{s l_{N}}\right)$. We can also bosonize the grading operator $d$. $d$ is defined by the property for the Chevalley generators,

$$
\left[d, t_{i}\right]=0, \quad\left[d, e_{i}^{ \pm}\right]= \pm \delta_{i 0} e_{i}^{ \pm}
$$

or equivalently, for the Drinfeld generators,

$$
\left[d, H_{n}^{i}\right]=n H_{n}^{i}, \quad\left[d, E_{n}^{ \pm, i}\right]=n E_{n}^{ \pm, i}
$$

Using eqs. 2.55) and (4.25), let us define the $q$-analogue of the Virasoro $L_{0}$ operator [17, 18] as follows:

$$
\begin{aligned}
L_{0} \stackrel{\text { def }}{=} & \frac{1}{2} \sum_{i, j=1}^{N-1} \sum_{n \in \mathbb{Z}}: a_{-n}^{i} \frac{n^{2}}{[n][(k+g) n]} \frac{[\min (i, j) n][(N-\max (i, j)) n]}{[N n][n]} a_{n}^{j}:+\sum_{i, j=1}^{N-1} \bar{\rho}^{i} \frac{a_{i j}^{-1}}{k+g} \hat{p}_{a}^{j} \\
& +\frac{1}{2} \sum_{1 \leq i<j \leq N} \sum_{n \in \mathbb{Z}}: b_{-n}^{i j}(-1) \frac{n^{2}}{[n]^{2}} b_{n}^{i j}:+\frac{1}{2} \sum_{1 \leq i<j \leq N} \hat{p}_{b}^{i j} \\
& +\frac{1}{2} \sum_{1 \leq i<j \leq N} \sum_{n \in \mathbb{Z}}: c_{-n}^{i j} \frac{n^{2}}{[n]^{2}} c_{n}^{i j}:+\frac{1}{2} \sum_{1 \leq i<j \leq N} \hat{p}_{c}^{i j},
\end{aligned}
$$

where $\bar{\rho}^{i}=1$, i.e., $\bar{\rho}=(1,1, \cdots, 1)=\sum_{i=1}^{N-1} \bar{\Lambda}_{i}$ is the half sum of positive roots of $s l_{N}$. Then $d=-L_{0}$ satisfies eq.(5.2) on the representation space given in Cor.77. The $L_{0}$ eigenvalue of $\left|\left(\ell^{1}, \cdots, \ell^{N-1}\right), 0,0\right\rangle$ is $\frac{1}{2(k+g)} \ell^{i} a_{i j}^{-1}\left(\ell^{j}+2 \bar{\rho}^{j}\right)=\frac{1}{2(k+g)}(\bar{\lambda}, \bar{\lambda}+2 \bar{\rho})$.

We have also constructed the screening currents and the vertex operators. Using these, we can start the representation theory and calculation of correlation functions. Like as $\widehat{s l_{N}}$ [28, 29], it is expected that the projection from the boson Fock space to the irreducible $U_{q}\left(\widehat{s l_{N}}\right)$ representation space can be done by BRST cohomology technique. In fact, recently, this procedure has been worked out for $U_{q}\left(\widehat{s l_{2}}\right)$ 30. The BRST operator is constructed by using the screening current.

To calculate the Jackson integral formulas for the correlation functions, which are solutions of $q$-KZ equation, we must first prepare the $q$-vertex operators $\Phi$. We will restrict ourselves to the type I [10] vertex operator $\Phi_{V(\mu)}^{V(\nu) V_{\lambda}}(z): V(\mu) \rightarrow V(\nu) \otimes V_{\lambda z}$. $\Phi_{V(\mu)}^{V(\nu) V_{\lambda}}(z)$ can be constructed from $\phi^{\bar{\lambda}}\left(q^{\beta} z ; \alpha\right)$ with appropriate $\alpha, \beta$. From eq.(4.24), we choose $\alpha=-\frac{k+g}{2}$. This choice agrees with refs. 31 $\left(U_{q}\left(\widehat{s l_{2}}\right)\right.$ with an arbitrary level $\left.k\right)$ and 32 (vector representation of $U_{q}\left(\widehat{s l_{N}}\right)$ with $\left.k=1\right)$. Starting from $\phi^{\bar{\lambda}}(z) \stackrel{\text { def }}{=} \phi^{\bar{\lambda}}\left(z ;-\frac{k+g}{2}\right)$, we define $\phi_{i_{1}, \cdots, i_{n}}^{\bar{\lambda}}(z)$ as follows:

$$
\phi_{i_{1}, \cdots, i_{n}}^{\bar{\lambda}}(z) \stackrel{\text { def }}{=}\left[\phi_{i_{1}, \cdots, i_{n-1}}^{\bar{\lambda}}(z), E_{0}^{-, i_{n}}\right]_{q^{x}}, \quad x=\left(\bar{\lambda}-\sum_{j=1}^{n-1} \bar{\alpha}_{i_{j}}, \bar{\alpha}_{i_{n}}\right) .
$$


To determine $\beta$, we must the specify finite dimensional representation of $U_{q}\left(\widehat{s l_{N}}\right)$. Results of refs. 31, 32 suggest $\beta=k+g$. Once the finite dimensional representation is obtained and $\beta$ is determined, we can construct the $q$-vertex operator $\Phi_{V(\mu)}^{V(\nu) V_{\lambda}}(z)$ from our $\phi_{i_{1}, \cdots, i_{n}}^{\bar{\lambda}}\left(q^{\beta} z\right)$. Then, we can calculate correlation functions of the $q$-vertex operators in standard way. These problems are now under investigation.

To extend our results to arbitrary quantum affine Lie algebras, it may be important to consider the geometrical interpretation of the free boson realization. For $q=1$ case, the $\beta-\gamma$ system is suitable for the geometrical interpretation 17. For $q \neq 1$ case, we define the quantum $\beta-\gamma$ fields, $\beta_{\alpha, \pm}^{i j}(z)(\alpha= \pm 1), \gamma^{i j}(z)$, as follows:

$$
\begin{aligned}
\beta_{1, \pm}(z) & \stackrel{\text { def }}{=} \frac{-1}{\left(q-q^{-1}\right) z}: \exp \left(b_{ \pm}(z)-(b+c)\left(q^{ \pm 1} z\right)\right): \\
\beta_{-1, \pm}(z) & \stackrel{\text { def }}{=} \frac{-1}{\left(q-q^{-1}\right) z}: \exp \left(-b_{\mp}(z)-(b+c)\left(q^{ \pm 1} z\right)\right): \\
\gamma(z) & \stackrel{\text { def }}{=}: \exp ((b+c)(z)):
\end{aligned}
$$

where we suppress the superscript $i j$. They are not free fields any longer. They satisfy

$$
\begin{aligned}
\left(z-q^{\alpha+\alpha^{\prime}} w\right) \beta_{\alpha, \epsilon}(z) \beta_{\alpha^{\prime}, \epsilon^{\prime}}(w) & =\left(q^{\alpha+\alpha^{\prime}} z-w\right) \beta_{\alpha^{\prime}, \epsilon^{\prime}}(w) \beta_{\alpha, \epsilon}(z) \quad\left(\epsilon, \epsilon^{\prime}= \pm\right), \\
\beta_{ \pm 1, \pm}(z) \beta_{\mp 1, \pm}(w) & =\beta_{\mp 1, \pm}(w) \beta_{ \pm 1, \pm}(z) \\
\left(z-q^{\mp 1} w\right) \beta_{ \pm 1, \pm}(z) \gamma(w) & =\left(q^{\mp 1} z-w\right) \gamma(w) \beta_{ \pm 1, \pm}(z) \\
\left(z-q^{\mp 1} w\right) \gamma(z) \beta_{ \pm 1, \mp}(w) & =\left(q^{\mp 1} z-w\right) \beta_{ \pm 1, \mp}(w) \gamma(z) \\
\gamma(z) \gamma(w) & =\gamma(w) \gamma(z) .
\end{aligned}
$$

Our free boson realization of $U_{q}\left(\widehat{s l_{N}}\right)$ is reexpressed by these quantum $\beta$ - $\gamma$ fields. In the $q \rightarrow 1$ limit, $\beta_{\alpha,+}(z)-\beta_{\alpha,-}(z)$ and $\gamma(z)$ become usual $\beta(z)$ and $\gamma(z)$ respectively. These $\beta_{\alpha, \pm}, \gamma$ fields are the affinization of $q$-oscillator $\left(a a^{\dagger}-q^{ \pm 1} a^{\dagger} a=q^{\mp \mathcal{N}}\right) ; a \rightarrow \gamma$, $a^{\dagger} \rightarrow \beta_{\alpha,+}-\beta_{\alpha,-}$ (see Appendix A). We expect that our realization in terms of the quantum $\beta-\gamma$ system acts on the $q$-deformed semi-infinite flag manifold [17].

Our free boson realization may be also useful to analyze the $q$-analogue of the Virasoro and $W$ algebras by the Hamiltonian reduction, and the representation at the critical level $k=-g$.

\section{Acknowledgments}

We would like to thank T. Inami for a careful reading the manuscript and discussions. We would also like to thank T. Eguchi, E. Frenkel, M. Jimbo, T. Miwa, A. Nakayashiki, M. Noumi, and Y. Yamada for helpful discussions. 


\section{Appendix A}

For the reader's convenience, we give the result of [25], the Heisenberg realization of $U_{q}\left(s l_{N}\right)$ with the weight $\lambda_{i} \in \mathbb{C}$. Let us consider variables $x_{i j}$ and derivatives $\frac{\partial}{\partial x_{i j}}(1 \leq$ $i<j \leq N)$. Their commutation relations are

$$
\left[\frac{\partial}{\partial x_{i j}}, x_{i^{\prime} j^{\prime}}\right]=\delta_{i i^{\prime}} \delta_{j j^{\prime}}, \quad \text { others }=0 .
$$

Standard Chevalley generators of $U_{q}\left(s l_{N}\right), e_{i}^{ \pm}, t_{i}=q^{h_{i}}(i=1, \cdots, N-1)$, are realized as follows:

$$
\begin{aligned}
h_{i} \stackrel{\text { def }}{=} & -\sum_{j=1}^{i}\left(\vartheta_{j, i+1}-\vartheta_{j, i}\right)+\lambda_{i}-\sum_{j=i+1}^{N}\left(\vartheta_{i, j}-\vartheta_{i+1, j}\right), \\
e_{i}^{+} \stackrel{\text { def }}{=} & \sum_{j=1}^{i} x_{j, i} \frac{1}{x_{j, i+1}}\left[\vartheta_{j, i+1}\right] q^{-\sum_{\ell=1}^{j-1}\left(\vartheta_{\ell, i+1}-\vartheta_{\ell, i}\right)} \\
e_{i}^{-} \stackrel{\text { def }}{=} & \sum_{j=1}^{i-1} x_{j, i+1} \frac{1}{x_{j, i}}\left[\vartheta_{j, i}\right] q^{\sum_{\ell=j+1}^{i}\left(\vartheta_{\ell, i+1}-\vartheta_{\ell, i}\right)-\lambda_{i}+\sum_{\ell=i+1}^{N}\left(\vartheta_{i, \ell}-\vartheta_{i+1, \ell}\right)} \\
& +x_{i, i+1}\left[\lambda_{i}-\sum_{\ell=i+1}^{N}\left(\vartheta_{i, \ell}-\vartheta_{i+1, \ell}\right)\right] \\
& -\sum_{j=i+2}^{N} x_{i, j} \frac{1}{x_{i+1, j}}\left[\vartheta_{i+1, j}\right] q^{\lambda_{i}-\sum_{\ell=j}^{N}\left(\vartheta_{i, \ell}-\vartheta_{i+1, \ell}\right)},
\end{aligned}
$$

where $\vartheta_{i j} \stackrel{\text { def }}{=} x_{i j} \frac{\partial}{\partial x_{i j}}, x_{i i} \stackrel{\text { def }}{=} 1, \vartheta_{i i} \stackrel{\text { def }}{=} 0$.

Our free field realization of $U_{q}\left(\widehat{s l_{N}}\right)$ is obtained by the following replacement with suitable argument:

$$
\begin{aligned}
x & \mapsto e^{(b+c)(z)} \\
-\vartheta & \mapsto \pm b_{ \pm}(z) \\
\lambda & \mapsto \pm a_{ \pm}(z) \\
{[A(z)] } & \mapsto \frac{e^{A_{+}(z)}-e^{A_{-}(z)}}{\left(q-q^{-1}\right) z} .
\end{aligned}
$$

\section{Appendix B}

In this appendix, we reexpress eqs. (3.4),(3.6), (3.7) and (4.1) by using the $q$-difference operator. These expressions are not unique and we give one of them.

Using the following formulas

$$
\frac{1}{\left(q-q^{-1}\right) z}\left(a_{+}\left(q^{\alpha} z\right)-a_{-}\left(q^{-\alpha} z\right)\right)={ }_{1} \partial_{z} a(z ; \alpha)=\sum_{n \in \mathbb{Z}} a_{n} q^{-\alpha|n|} z^{-n-1},
$$




$$
\begin{aligned}
& \frac{1}{\left(q-q^{-1}\right) z}:\left(\exp \left( \pm b_{ \pm}(z)-(b+c)(q z)\right)-\exp \left( \pm b_{\mp}(z)-(b+c)\left(q^{-1} z\right)\right)\right): \\
& \quad=:{ }_{1} \partial_{z}(\exp (-c(z))) \cdot \exp (-b(z ; \mp 1)):, \\
& \begin{aligned}
\frac{1}{\left(q-q^{-1}\right) z} & :\left(\exp \left( \pm a_{+}\left(q^{\alpha} z\right)\right)-\exp \left( \pm a_{-}\left(q^{-\alpha} z\right)\right)\right): \\
& =:{ }_{M} \partial_{z}\left(\exp \left( \pm\left(\frac{1}{M} a\right)(z ; \alpha)\right)\right) \cdot \exp \left(\mp\left(\frac{1}{M} a\right)(z ; \alpha-M)\right): \\
\frac{1}{\left(q-q^{-1}\right) z} & :\left(\exp \left(b\left(q^{\alpha} z\right)\right)-\exp \left(b\left(q^{-\alpha} z\right)\right)\right): \\
& =:{ }_{M} \partial_{z}\left(\exp \left(\left(\frac{\alpha}{M} b\right)(z)\right)\right) \cdot \exp \left(\left(\frac{M-\alpha}{M} b\right)(z)\right):
\end{aligned} \\
& \left(\frac{\alpha}{M} b\right)(z) \pm\left(\frac{1}{M} b\right)(z ; \pm \alpha+1)=\left(\frac{\alpha \pm 1}{M} b\right)(z ; 1),
\end{aligned}
$$

eqs.(3.4),(3.6), (3.7) and (4.1) are rewritten as follows:

$$
\begin{aligned}
& H^{i}(z)={ }_{1} \partial_{z}\left(\sum_{j=1}^{i}\left(b^{j, i+1}\left(z ; \frac{k}{2}+j-1\right)-b^{j, i}\left(z ; \frac{k}{2}+j\right)\right)\right. \\
&\left.+a^{i}\left(z ; \frac{g}{2}\right)+\sum_{j=i+1}^{N}\left(b^{i, j}\left(z ; \frac{k}{2}+j\right)-b^{i+1, j}\left(z ; \frac{k}{2}+j-1\right)\right)\right) \\
& E^{+, i}(z)=-\sum_{j=1}^{i}: \exp \left((b+c)^{j, i}\left(q^{j-1} z\right)\right) \\
& \times{ }_{1} \partial_{z}\left(\exp \left(-c^{j, i+1}\left(q^{j-1} z\right)\right)\right) \cdot \exp \left(-b^{j, i+1}\left(q^{j-1} z ;-1\right)\right) \\
& \times \exp \left(\sum_{\ell=1}^{j-1}\left(b_{+}^{\ell, i+1}\left(q^{\ell-1} z\right)-b_{+}^{\ell, i}\left(q^{\ell} z\right)\right)\right): \\
& E^{-, i}(z)=-\sum_{j=1}^{i-1}: \exp \left((b+c)^{j, i+1}\left(q^{-(k+j)} z\right)\right) \\
& \quad \times{ }_{1} \partial_{z}\left(\exp \left(-c^{j i}\left(q^{-(k+j)} z\right)\right)\right) \cdot \exp \left(-b^{j i}\left(q^{-(k+j)} z ; 1\right)\right) \\
& \quad \times \exp \left(\sum_{\ell=j+1}^{i}\left(b_{-}^{\ell, i+1}\left(q^{-(k+\ell-1)} z\right)-b_{-}^{\ell, i}\left(q^{-(k+\ell)} z\right)\right)\right. \\
&\left.\quad+a_{-}^{i}\left(q^{-\frac{k+g}{2}} z\right)+\sum_{\ell=i+1}^{N}\left(b_{-}^{i, \ell}\left(q^{-(k+\ell)} z\right)-b_{-}^{i+1, \ell}\left(q^{-(k+\ell-1)} z\right)\right)\right): \\
&+ \\
& \quad{ }_{k+g} \partial_{z}\left(\operatorname { e x p } \left(\left(\frac{k+i}{k+g}(b+c)^{i, i+1}\right)(z)+\left(\frac{1}{k+g} a^{i}\right)\left(z ; \frac{k+g}{2}\right)\right.\right. \\
&\left.\left.\quad+\sum_{\ell=i+1}^{N}\left(\left(\frac{1}{k+g} b^{i, \ell}\right)(z ; k+\ell)-\left(\frac{1}{k+g} b^{i+1, \ell}\right)(z ; k+\ell-1)\right)\right)\right) \\
& \quad \times \exp \left(\left(\frac{g-i}{k+g}(b+c)^{i, i+1}\right)(z)-\left(\frac{1}{k+g} a^{i}\right)\left(z ;-\frac{k+g}{2}\right)\right.
\end{aligned}
$$




$$
\begin{aligned}
& \left.-\sum_{\ell=i+1}^{N}\left(\left(\frac{1}{k+g} b^{i, \ell}\right)(z ; \ell-g)-\left(\frac{1}{k+g} b^{i+1, \ell}\right)(z ; \ell-g-1)\right)\right): \\
+\sum_{j=i+2}^{N} & : \exp \left((b+c)^{i, j}\left(q^{k+j-1} z\right)\right) \\
& \times{ }_{1} \partial_{z}\left(\exp \left(-c^{i+1, j}\left(q^{k+j-1} z\right)\right)\right) \cdot \exp \left(-b^{i+1, j}\left(q^{k+j-1} z ;-1\right)\right) \\
& \times \exp \left(a_{+}^{i}\left(q^{\frac{k+g}{2}} z\right)+\sum_{\ell=j}^{N}\left(b_{+}^{i, \ell}\left(q^{k+\ell} z\right)-b_{+}^{i+1, \ell}\left(q^{k+\ell-1} z\right)\right)\right): \\
S^{i}(z)=- & \left.\exp ^{k+}\left(-\frac{1}{k+g} a^{i}\right)\left(z ; \frac{k+g}{2}\right)\right) \\
\times \sum_{j=i+1}^{N} & \exp \left((b+c)^{i+1, j}\left(q^{N-j} z\right)\right) \\
\quad & { }_{1} \partial_{z}\left(\exp \left(-c^{i, j}\left(q^{N-j} z\right)\right)\right) \cdot \exp \left(-b^{i, j}\left(q^{N-j} z ; 1\right)\right) \\
& \times \exp \left(\sum_{\ell=j+1}^{N}\left(b_{-}^{i+1, \ell}\left(q^{N-\ell+1} z\right)-b_{-}^{i, \ell}\left(q^{N-\ell} z\right)\right)\right):
\end{aligned}
$$

These expressions are adequate for taking the $q \rightarrow 1$ limit, because there is no denominator $q-q^{-1}$. In this limit ${ }_{\alpha} \partial_{z},\left(\frac{L_{1}}{M_{1}} \cdots \frac{L_{r}}{M_{r}} a\right)(z ; \alpha),\left(\frac{L_{1}}{M_{1}} \cdots \frac{L_{r}}{M_{r}} a_{ \pm}\right)(z)$ become $\alpha \partial_{z}$, $\frac{L_{1} \cdots L_{r}}{M_{1} \cdots M_{r}} a(z), 0$ respectively. Eqs.(3.4), (3.6) and (3.7) become the bosonized version of the Wakimoto realization of $\widehat{s l_{N}}$ with level $k\left[16\right.$, 17, 18]; $\beta^{i j}(z)$ and $\gamma^{i j}(z)$ are expressed in terms of $b^{i j}(z)$ and $c^{i j}(z)$ with $q=1$ as follows [33]:

$$
\begin{aligned}
& \beta^{i j}(z)=-: \partial_{z}\left(\exp \left(-c^{i j}(z)\right)\right) \cdot \exp \left(-b^{i j}(z)\right): \\
& \gamma^{i j}(z)=: \exp \left((b+c)^{i j}(z)\right):
\end{aligned}
$$

\section{Appendix $\mathrm{C}$}

In this appendix we give useful formulas.

First we give formulas for a boson $a$ in section 2 (see the footnote below eq.(2.19)) .

$$
\begin{gathered}
{[A, B] \text { commute with } A, B \Rightarrow\left[A, e^{B}\right]=[A, B] e^{B},} \\
e^{A} e^{B}=e^{[A, B]} e^{B} e^{A} \\
{\left[a_{n},\left(\frac{L_{1}}{M_{1}} \cdots \frac{L_{r}}{M_{r}} a_{ \pm}\right)(z)\right]= \pm \theta(\mp n>0)\left(q-q^{-1}\right) \frac{\left[L_{1} n\right] \cdots\left[L_{r} n\right]}{\left[M_{1} n\right] \cdots\left[M_{r} n\right]} n \rho_{a}(n) z^{n},} \\
{\left[a_{n},\left(\frac{L_{1}}{M_{1}} \cdots \frac{L_{r}}{M_{r}} a\right)(z ; \alpha)\right]=\frac{\left[L_{1} n\right] \cdots\left[L_{r} n\right]}{\left[M_{1} n\right] \cdots\left[M_{r} n\right]} \frac{n}{[n]} \rho_{a}(n) q^{-\alpha|n|} z^{n}}
\end{gathered}
$$




$$
\begin{aligned}
& {\left[\left(\frac{L_{1}}{M_{1}} \cdots \frac{L_{r}}{M_{r}} a_{+}\right)(z),\left(\frac{L_{1}^{\prime}}{M_{1}^{\prime}} \cdots \frac{L_{s}^{\prime}}{M_{s}^{\prime}} a_{-}\right)(w)\right]} \\
& =-\left(q-q^{-1}\right)^{2} \sum_{n>0} \frac{\left[L_{1} n\right] \cdots\left[L_{r} n\right]}{\left[M_{1} n\right] \cdots\left[M_{r} n\right]} \frac{\left[L_{1}^{\prime} n\right] \cdots\left[L_{s}^{\prime} n\right]}{\left[M_{1}^{\prime} n\right] \cdots\left[M_{s}^{\prime} n\right]} n \rho_{a}(n)\left(\frac{w}{z}\right)^{n}, \quad(\mathrm{C} .5) \\
& {\left[\left(\frac{L_{1}}{M_{1}} \cdots \frac{L_{r}}{M_{r}} a\right)(z ; \alpha),\left(\frac{L_{1}^{\prime}}{M_{1}^{\prime}} \cdots \frac{L_{s}^{\prime}}{M_{s}^{\prime}} a_{-}\right)(w)\right]} \\
& =\left(q-q^{-1}\right) \sum_{n>0} \frac{\left[L_{1} n\right] \cdots\left[L_{r} n\right]}{\left[M_{1} n\right] \cdots\left[M_{r} n\right]} \frac{\left[L_{1}^{\prime} n\right] \cdots\left[L_{s}^{\prime} n\right]}{\left[M_{1}^{\prime} n\right] \cdots\left[M_{s}^{\prime} n\right]} \frac{n}{[n]} \rho_{a}(n) q^{-\alpha n}\left(\frac{w}{z}\right)^{n} \\
& \quad+\frac{L_{1} \cdots L_{r}}{M_{1} \cdots M_{r}} \frac{L_{1}^{\prime} \cdots L_{s}^{\prime}}{M_{1}^{\prime} \cdots M_{s}^{\prime}} \rho_{a} \log q \\
& {\left[\left(\frac{L_{1}^{\prime}}{M_{1}^{\prime}} \cdots \frac{L_{s}^{\prime}}{M_{s}^{\prime}} a_{+}\right)(z),\left(\frac{L_{1}}{M_{1}} \cdots \frac{L_{r}}{M_{r}} a\right)(w ; \alpha)\right]=\mathrm{eq} \cdot(\mathrm{C} \cdot 6)} \\
& {\left[\left(\frac{L_{1}}{M_{1}} \cdots \frac{L_{r}}{M_{r}} a\right)(z ; \alpha),\left(\frac{L_{1}^{\prime}}{M_{1}^{\prime}} \cdots \frac{L_{s}^{\prime}}{M_{s}^{\prime}} a\right)(w ; \beta)\right]} \\
& =-\sum_{n \neq 0} \frac{\left[L_{1} n\right] \cdots\left[L_{r} n\right]}{\left[M_{1} n\right] \cdots\left[M_{r} n\right]} \frac{\left[L_{1}^{\prime} n\right] \cdots\left[L_{s}^{\prime} n\right]}{\left[M_{1}^{\prime} n\right] \cdots\left[M_{s}^{\prime} n\right]} \frac{n}{[n]^{2}} \rho_{a}(n) q^{-(\alpha+\beta)|n|}\left(\frac{w}{z}\right)^{n} \\
& \quad-\frac{L_{1} \cdots L_{r}}{M_{1} \cdots M_{r}} \frac{L_{1}^{\prime} \cdots L_{s}^{\prime}}{M_{1}^{\prime} \cdots M_{s}^{\prime}} \rho_{a} \log \frac{w}{z}
\end{aligned}
$$

where $\theta(P)$ is a step function, $\theta(P)=1(0)$ when the proposition $P$ is true(false). These are formal power series equations.

Next we give specific formulas often used in proofs. For calculation of $\left[H_{n}^{i}, *\right]$,

$$
\begin{aligned}
{\left[a_{n}^{i}, a_{ \pm}^{j}(z)\right] } & = \pm \theta(\mp n>0)\left(q-q^{-1}\right) \frac{1}{n}[(k+g) n]\left[a_{i j} n\right] z^{n}, \\
{\left[a_{n}^{i},\left(\frac{1}{k+g} a^{j}\right)(z ; \alpha)\right] } & =\frac{1}{n}\left[a_{i j} n\right] q^{-\alpha|n|} z^{n} \\
{\left[b_{n}, b_{ \pm}(z)\right] } & =\mp \theta(\mp n>0)\left(q-q^{-1}\right) \frac{1}{n}[n]^{2} z^{n} \\
{\left[b_{n}, b(z)\right] } & =-\frac{1}{n}[n] z^{n}
\end{aligned}
$$

where we suppress the superscript of $b^{i j}$. For OPE calculation,

$$
\begin{aligned}
\exp \left(\alpha b_{+}(z)\right) \exp \left(\beta b_{-}(w)\right) & \left(\frac{(z-w)^{2}}{\left(z-q^{2} w\right)\left(z-q^{-2} w\right)}\right)^{\alpha \beta} \exp \left(\beta b_{-}(w)\right) \exp \left(\alpha b_{+}(z)\right) \\
\exp \left(\alpha b_{+}(z)\right): \exp (\beta b(w)): & \simeq\left(\frac{z-q w}{q z-w}\right)^{\alpha \beta}: \exp (\beta b(w)): \exp \left(\alpha b_{+}(z)\right), \\
: \exp (\alpha b(z)): \exp \left(\beta b_{-}(w)\right) & \simeq\left(\frac{z-q w}{q z-w}\right)^{\alpha \beta} \exp \left(\beta b_{-}(w)\right): \exp (\alpha b(z)): \\
& =\left(\frac{z-q w}{q z-w}\right)^{\alpha \beta} q^{\alpha \beta}: \exp \left(\alpha b(z)+\beta b_{-}(w)\right):
\end{aligned}
$$




$$
\begin{aligned}
& \exp \left(a_{+}^{i}\left(q^{\frac{k+g}{2}} z\right)\right) \exp \left(a_{-}^{j}\left(q^{-\frac{k+g}{2}} w\right)\right) \\
\simeq & \frac{z-q^{a_{i j}} w}{z-q^{-a_{i j}} w} \frac{z-q^{-a_{i j}-2(k+g)} w}{z-q^{a_{i j}-2(k+g)} w} \exp \left(a_{-}^{j}\left(q^{-\frac{k+g}{2}} w\right)\right) \exp \left(a_{+}^{i}\left(q^{\frac{k+g}{2}} z\right)\right), \\
& \exp \left(a_{+}^{i}\left(q^{\frac{k+g}{2}} z\right)\right): \exp \left(-\left(\frac{1}{k+g} a^{j}\right)\left(w ; \frac{k+g}{2}\right)\right): \\
\simeq & \frac{z-q^{a_{i j}-(k+g)} w}{q^{a_{i j}} z-q^{-(k+g)} w}: \exp \left(-\left(\frac{1}{k+g} a^{j}\right)\left(w ; \frac{k+g}{2}\right)\right): \exp \left(a_{+}^{i}\left(q^{\frac{k+g}{2}} z\right)\right), \\
& : \exp \left(-\left(\frac{1}{k+g} a^{i}\right)\left(z ; \frac{k+g}{2}\right)\right): \exp \left(a_{-}^{j}\left(q^{-\frac{k+g}{2}} w\right)\right) \\
\simeq & \frac{z-q^{a_{i j}-(k+g)} w}{q^{a_{i j}} z-q^{-(k+g)} w} \exp \left(a_{-}^{j}\left(q^{-\frac{k+g}{2}} w\right)\right): \exp \left(-\left(\frac{1}{k+g} a^{i}\right)\left(z ; \frac{k+g}{2}\right)\right): \\
= & \frac{z-q^{a_{i j}-(k+g)} w}{q^{a_{i j}} z-q^{-(k+g)} w} q^{a_{i j}}: \exp \left(-\left(\frac{1}{k+g} a^{i}\right)\left(z ; \frac{k+g}{2}\right)+a_{-}^{j}\left(q^{-\frac{k+g}{2}} w\right)\right):,
\end{aligned}
$$

where $\alpha$ and $\beta$ are parameters and $\simeq$ means equality in the OPE sense (analytic continuation sense).

$\exp (b+c)$ 's commute each other because $\rho_{b}(n)+\rho_{c}(n)=0$.

\section{Appendix D}

In this appendix we give how poles cancel each other in OPE of $E^{+, i}(z)$ and $E^{-, j}(w)$, $E^{-, i}(z)$ and $E^{-, j}(w), E^{ \pm, i}(z)$ and $S^{j}(w)$. Let us denote each term of eqs.(3.6),(3.7),(4.1) as follows]:

$$
\begin{aligned}
E^{+, i}(z)= & \sum_{j=1}^{i}\left(E^{+, i(j, 1)}(z)+E^{+, i(j, 2)}(z)\right), \\
E^{-, i}(z)= & \sum_{j=1}^{i-1}\left(E^{-, i(j, 1)}(z)+E^{-, i(j, 2)}(z)\right) \\
& +E^{-, i(i, 1)}(z)+E^{-, i(i, 2)}(z)+\sum_{j=i+2}^{N}\left(E^{-, i(j, 1)}(z)+E^{-, i(j, 2)}(z)\right), \\
S^{i}(z)= & \sum_{j=i+1}^{N}\left(S^{i(j, 1)}(z)+S^{i(j, 2)}(z)\right) .
\end{aligned}
$$

I. $E^{+, i}(z) E^{-, j}(w)$.

For $i=j$, OPE $E^{+, i}(z) E^{-, j}(w)$ has poles at $z=q^{k} w$ and $z=q^{-k} w$. They come from $E^{+, i(i, 1)}(z) E^{-, j(j, 2)}(w)$ and $E^{+, i(1,2)}(z) E^{-, j(1,1)}(w)$ respectively.

${ }^{8}$ For example, $E^{+, i(j, 2)}(z)=\frac{-1}{\left(q-q^{-1}\right) z}: \exp \left((b+c)^{j, i}\left(q^{j-1} z\right)\right) \times(-1) \exp \left(b_{-}^{j, i+1}\left(q^{j-1} z\right)-(b+\right.$ $\left.c)^{j, i+1}\left(q^{j-2} z\right)\right) \times \exp \left(\sum_{\ell=1}^{j-1}\left(b_{+}^{\ell, i+1}\left(q^{\ell-1} z\right)-b_{+}^{\ell, i}\left(q^{\ell} z\right)\right)\right):$. 
Some terms of $E^{+, i}(z) E^{-, j}(w)$ have other poles but all these poles cancel in pairs. We give these poles $\left(z=q^{\alpha} w\right)$ and pairs $\left(E^{+, i(A)}(z) E^{-, j(B)}(w)\right.$ and $\left.E^{+, i(C)}(z) E^{-, j(D)}(w)\right)$.

$$
\begin{aligned}
& \begin{array}{lllll}
\alpha & (A) & (B) & (C) & (D)
\end{array} \\
& \text { (i) } j=i \quad-k-2 \ell \quad(\ell, 1) \quad(\ell, 2) \quad(\ell+1,2) \quad(\ell+1,1) \\
& \text { (ii) } j=i+1 \quad-k-2 \ell+1 \quad(\ell, 1) \quad(\ell, 2) \quad(\ell, 2) \quad(\ell, 1) \\
& \text { (iii) } j=i-1 \quad k+1 \quad(i-1,1) \quad(j+2,1) \quad(i, 1) \quad(j, 2) \\
& k+1 \quad(i-1,1) \quad(j+2,2) \quad(i, 2) \quad(j, 2) \\
& k+1 \quad(i, 1) \quad(j+2,2) \quad(i, 2) \quad(j+2,1) \\
& \text { (iv) } j \leq i-2 \quad k+i-j \quad(j, 1) \quad(i+1,1) \quad(j+1,1) \quad(i, 1) \\
& k+i-j \quad(j, 1) \quad(i+1,2) \quad(j+1,2) \quad(i, 1) \\
& k+i-j \quad(j+1,1) \quad(i+1,2) \quad(j+1,2) \quad(i+1,1) \text {. }
\end{aligned}
$$

II. $E^{-, i}(z) E^{-, j}(w)$.

$E^{-, i}(z) E^{-, j}(w)$ has poles at $z=q^{-a_{i j}} w$. Some terms of this OPE have extra poles. But these extra poles $\left(z=q^{\alpha} w\right)$ cancel in the following pairs $\left(E^{-, i(A)}(z) E^{-, j(B)}(w)\right.$ and $\left.E^{-, i(C)}(z) E^{-, j(D)}(w)\right)$.

$$
\begin{aligned}
& \begin{array}{llll}
\alpha & (A) \quad(B) & (C) \quad(D)
\end{array} \\
& \text { (i) } j=i-1 \quad 2 k+i+j \quad(i-1,2) \quad(j, 2) \quad(i, 1) \quad(j+2,2) \\
& \text { (ii) } j \leq i-2 \quad 2 k+i+j \quad(j, 2) \quad(i, 1) \quad(j+1,1) \quad(i+1,2) \\
& 2 k+i+j \quad(j, 2) \quad(i, 2) \quad(j+1,2) \quad(i+1,2) \\
& 2 k+i+j \quad(j+1,2) \quad(i, 1) \quad(j+1,1) \quad(i, 2) .
\end{aligned}
$$

III. $E^{+, i}(z) S^{j}(w)$.

Poles $\left(z=q^{\alpha} w\right)$ cancel in the following pairs $\left(E^{+, i(A)}(z) S^{j(B)}(w)\right.$ and $\left.E^{+, i(C)}(z) S^{j(D)}(w)\right)$.

$$
\begin{aligned}
& \begin{array}{lllll}
\alpha & (A) & (B) & (C)
\end{array} \\
& \text { (i) } j=i \quad N-i-j \quad(i, 1) \quad(j+1,2) \quad(i, 2) \quad(j+1,1) \\
& \text { (ii) } j \leq i-1 \quad N-i-j \quad(j, 1) \quad(i, 2) \quad(j+1,2) \quad(i+1,1) \\
& N-i-j \quad(j, 1) \quad(i+1,2) \quad(j, 2) \quad(i+1,1) \\
& N-i-j \quad(j, 2) \quad(i, 2) \quad(j+1,2) \quad(i+1,2) .
\end{aligned}
$$

IV. $E^{-, i}(z) S^{j}(w)$.

For $i=j$, OPE $E^{-, i}(z) S^{j}(w)$ has poles at $z=q^{k+g} w$ and $z=q^{-(k+g)} w$. They come from $E^{-, i(N, 1)}(z) S^{j(N, 2)}(w)$ and $E^{-, i(i, 1)}(z) S^{j(j+1,1)}(w)$ respectively.

Some terms of $E^{-, i}(z) S^{j}(w)$ have other poles but all these poles cancel in pairs. Poles $(z=$ 
$\left.q^{\alpha} w\right)$ and pairs $\left(E^{-, i(A)}(z) S^{j(B)}(w)\right.$ and $\left.E^{-, i(C)}(z) S^{j(D)}(w)\right)$ are
$\begin{array}{lll}\alpha & (A) & (B)\end{array}$
(C)
(D)
$\begin{array}{llllll}\text { (i) } \quad j=i & k-N+2 i+2 & (i, 2) & (j+1,2) & (i+2,2) & (j+2,1) \\ & k-N+2 \ell & (\ell, 1) & (\ell, 2) & (\ell+1,2) & (\ell+1,1)\end{array}$
$\begin{array}{llll}k-N+2 \ell \quad(\ell, 1) \quad(\ell, 2) \quad(\ell+1,2) & (\ell+1,1) \\ & & i+2 \leq \ell \leq N-1\end{array}$
(ii) $j=i+1 \quad k-N+2 \ell-1 \quad(\ell, 1) \quad(\ell, 2)$
$(\ell, 2)$
$(\ell, 1)$
$i+1 \leq \ell \leq N$
(iii) $j \leq i-$

$\begin{array}{lllll}-k-g+i-j & (j, 1) & (i+1,1) & (j+1,1) & (i, 1) \\ -k-g+i-j & (j, 2) & (i, 1) & (j, 1) & (i, 2) \\ -k-g+i-j & (j, 2) & (i+1,1) & (j+1,1) & (i, 2)\end{array}$




\section{References}

[1] A.A. Belavin, A.M. Polyakov and A.B. Zamolodchikov, Nucl. Phys. B241 (1984) 333-380.

[2] A.B. Zamolodchikov, Adv. Stud. Pure Math. 19 (1989) 641-674.

[3] T. Eguchi and S.-K. Yang, Phys. Lett. B224 (1989) 373-378; B235 (1990) 282-286.

[4] B.L. Feigin and E.V. Frenkel, Phys. Lett. B276 (1992) 79-86.

[5] D. Bernard and A. LeClair, Comm. Math. Phys. 142 (1991) 99-138.

[6] V.G. Knizhnik and A.B. Zamolodchikov, Nucl. Phys. B247 (1984) 83-103.

[7] A. Tsuchiya and Y. Kanie, Adv. Stud. Pure Math. 16 (1988) 297-372; Errata ibid. 19 (1989) 675-682.

[8] F.A. Smirnov, Int. J. Mod. Phys. A7 Suppl.1 (1992) 813-837;839-858.

[9] I.B. Frenkel and N.Yu. Reshetikhin, Comm. Math. Phys. 146 (1992) 1-60.

[10] B. Davies, O. Foda, M. Jimbo, T. Miwa and A. Nakayashiki, Comm. Math. Phys. 151 (1993) 89-153.

[11] B.L. Feigin and D.B. Fuchs, "Representations of the Virasoro Algebra", in Representations of infinite-dimensional Lie groups and Lie algebras, Gordon \& Breach 1986.

[12] Vl.S. Dotsenko and V.A. Fateev, Nucl. Phys. B240 [FS12] (1984) 312-348; B251 [FS13] (1985) 691-734.

[13] H. Awata, A. Tsuchiya and Y. Yamada, Nucl. Phys. B365 (1991) 680-696.

[14] M. Jimbo, K. Miki, T. Miwa and A. Nakayashiki, Phys. Lett. A168 (1992) 256-263.

[15] M. Idzumi, K. Iohara, M. Jimbo, T.Miwa, T. Nakashima and T. Tokihiro, Int. J. Mod. Phys. A8 (1993) 1479-1511.

[16] M. Wakimoto, Comm. Math. Phys. 104 (1986) 605-609.

[17] B.L. Feigin and E.V. Frenkel, Russian Math. Surveys 43 (1988) 221-222; Comm. Math. Phys. 128 (1990) 161-189.

[18] K. Ito and Y. Kazama, Mod. Phys. Lett. A5 (1990) 215-224;

M. Kuwahara and H. Suzuki, Phys. Lett. B235 (1990) 52-56. 
[19] I.B. Frenkel and N. Jing, Proc. Natl. Acad. Sci. USA 85 (1988) 9373-9377.

[20] J. Shiraishi, Phys. Lett. A171 (1992) 243-248.

[21] A. Matsuo, "Free Field Realization of Quantum Affine Algebra $U_{q}\left(\widehat{s l}_{2}\right)$ ", Nagoya Univ. preprint (1992); "Free Field Realization of $q$-Deformed Primary Fields for $U_{q}\left(\widehat{s l_{2}}\right) "$, Nagoya Univ. preprint (1992).

[22] A. Abada, A.H. Bougourzi adn M.A. El Gradechi, "A Deformation of the Wakimoto Construction", preprint CRM-1829 (1992).

[23] K. Kimura, "On Free Boson Representation of the Quantum Affine Algebra $U_{q}\left(\widehat{s l}_{2}\right)$ ", preprint RIMS-910 (1992);

A.H. Bougourzi, "Uniqueness of the Bosonization of the $U_{q}\left(s l(2)_{k}\right)$ Quantum Current Algebra", preprint CRM-1852 (1993).

[24] H. Awata, S. Odake and J. Shiraishi, "Free Boson Representation of $U_{q}\left(\widehat{s l}_{3}\right)$ ", preprint RIMS-920, YITP/K-1017 (1993).

[25] H. Awata, M. Noumi and S. Odake, "Heisenberg Realization for $U_{q}\left(s l_{n}\right)$ on the Flag Manifold", preprint YITP/K-1016 (1993).

[26] M. Jimbo, Lett. Math. Phys. 10 (1985) 63-69;

V.G. Drinfeld, Soviet Math. Dokl. 32 (1985) 254-258.

[27] V.G. Drinfeld, Soviet Math. Dokl. 36 (1988) 212-216.

[28] D. Bernard and G. Felder, Comm. Math. Phys. 127 (1990) 145-168.

[29] B.L. Feigin and E.V. Frenkel, in Physics and Mathematics of Strings, ed. L. Brink et al. (World Scientific, Singapore, 1990) p.271-316;

P. Bouwknegt, J. McCarthy and K. Pilch, Comm. Math. Phys. 131 (1990) 125-155.

[30] H. Konno, talk at the meeting of the Physical Society of Japan, March 1993.

[31] A. Kato, Y.-H. Quano and J. Shiraishi, "Free Boson Representation of $q$-Vertex Operators and their Correlation Functions", preprint UT-618 (1992), to appear in Comm. Math. Phys.

[32] Y. Koyama, "One-Point Functions of Vertex Models" (in Japanese), master thesis (RIMS, Kyoto Univ.) (1993).

[33] D. Friedan, E. Martinec and S. Shenker, Nucl. Phys. B271 (1986) 93-165. 